\section{Computing lag sequential statistics on dyadic time interval data: The TLAG program}

\author{
DOUGLAS K. SYMONS, RICHARD D. WRIGHT, \\ and GREG MORAN \\ University of Western Ontario, London, Ontario, Canada
}

Lag sequential statistics have been developed to assess contingency relationships within a series of behavioral codes (e.g., Allison \& Liker, 1982; Bakeman, 1983; Bakeman \& Gottman, 1986; Sackett, 1979; Sackett, Holm, Crowley, \& Henkins, 1979). A common way of representing ongoing dyadic behavior is to code behavioral states and/or actions during successive time intervals (see Sackett, 1979). The purpose of the present paper is to describe a program written in IBM-BASIC that produces lag sequential statistics on two concurrent streams of interval codes. The TLAG program was written because this type of data cannot easily be analyzed by existing FORTRAN programs (Bakeman, 1983; Sackett et al., 1979), which were designed to analyze single streams of behavioral codes and are more effectively used with event codes (see the ELAG program, Bakeman, 1983). Mother-infant interaction research will be used to illustrate the use of TLAG.

\section{General Capabilities}

Interactive behavior is often coded either as a sequence of discrete events without a real-time base (event coding) or as a sequence of occurrences/non-occurrences of behavior during adjacent time intervals (interval coding). Event coding incorporates actions by both interactants into a single stream. Interval coding of interactive behavior can also be reduced into a single stream, but this method is most effective when each interactant's behavior is coded as an independent behavior stream. Although both event coding and interval coding have advantages and limitations (see Altmann, 1974; Sackett, 1979; Simpson, 1979), interval coding retains both sequential and real-time information.

The focus in the analysis of social interaction, and therefore in the TLAG program, is the contingency or sequential dependency between the actions of two interactants. The first stage of this analysis is the transformation of the original interval-coded data set for each interactant into a stream of intervals coded as including either a change or no change in behavior. The definition of change/no change, and thus the nature of this transformation, is a function of the question at hand. For example, maternal imitation of infant smiling may be evaluated by reducing a complex set of codes to "smile/no smile." The original stream of codes remains available for further transformation suiting different purposes.

Address for correspondence is Department of Psychology, University of Western Ontario, London, ON N6A 5C2, Canada.
The logic of the TLAG program can be illustrated with an example from a recent analysis in a research program in which Symons and Moran (1987) described free-play interactions between mothers and their 3-month-old infants. Behavior in four categories was independently coded as occurring (1) or not occurring (0). Vocalizations, smiles, and gazes at the partner were coded for both interactants. In addition, a mother's touching of her infant was coded for the mother, and the infant's crying was coded for the infant. Action in each category was coded in each of $1801-\mathrm{sec}$ intervals over a 3-min episode of continuous interaction. A data file for one episode thus was composed of $1 \mathrm{~s}$ and $0 \mathrm{~s}$ in a matrix of 8 columns (4 mother and 4 infant behaviors per second) and 180 rows (1-sec intervals).

In the most straightforward analysis when only one code, smiling, was entered into the transformation, we found that onsets of smiling by an infant were followed by onsets of smiling by the mother more frequently than expected by chance. This pattern of maternal imitation occurred at a number of 1-sec lags and in several other categories of behavior (Moran, Krupka, Tutton, \& Symons, 1987). In a related study, we defined action for both interactants as a change in any coded category of behavior. The analyses revealed that both mothers and infants were behaviorally responsive to changes in behavior by their social partners (Symons \& Moran, 1987). This analysis was derived from the version of TLAG presented in this article.

There has been recent discussion regarding the details of the statistic best suited to sequential analysis (see Allison \& Liker, 1982; Bakeman, 1983). TLAG calculates $z$ statistics for each subject-pair as the square root of the chi-square statistic for a $2 \times 2$ contingency table (see Dumas, 1986) of the change/no change in behavior of each of two interactants. This yields normalized values equal to those derived according to the formula proposed by Allison and Liker (1982). TLAG also calculates a mean $z$ statistic for the sample, because any population of $z$ scores has an expected mean of 0 and a standard deviation of 1 , and this mean can be tested for significant deviation from the expected mean $z$ score for a sample of a given size (as suggested by Sackett, 1979, p. 645). This is a conservative way of utilizing sequential analyses with groups of subject-pairs when the contingency tables have similar unconditional base rates of behavior and total number of observations (Moran \& Symons, 1987). However, there is some concern about heterogeneity of Markov chains of behavior across dyads (see Gardner, 1987).

Gardner and Hartmann (1984) have explained two kinds of dependency contained in the coded behavior streams of two interactants: dependency within the data of each interactant (serial dependency or autocorrelation) and dependency between the interactants' data (joint dependency or cross-correlation) (see also Allison \& Liker, 1982; 
Dumas, 1986; Gardner, Hartmann, \& Mitchell, 1982). The presence of autocorrelation can significantly affect measures of cross-correlation. Therefore, any assessment of cross-correlation must be corrected for the autocorrelation within each interactant's data. TLAG employs such a correction for first-order Markov autocorrelation (see Dumas, 1986; Gardner \& Hartmann, 1984).

In summary, the strengths of the current BASIC program are that (1) it reads parallel streams of multiple codes of dyadic time interval data, (2) it incorporates important controls for autocorrelation so that dependency between interactants can be accurately assessed, and (3) it is intended to be readily understood and modifiable by other users. The program is written in IBM-BASIC, but is easily convertible to other forms of BASIC and also requires very little computer memory to run. It can therefore be used with almost any type of computer system. Finally, the program can be converted to many types of contingency analyses by changing IF statements.

\section{Specific Capabilities}

TLAG is written in IBM-BASIC and functions by reading a data file for a dyad, calculating and saving sequential statistics, and then repeating all steps for subsequent dyads (see Listing 1).

Reading a Data File. Data is input in lines 100-120 using OPEN, READ, and INPUT statements from data files listed in line 90 . Each of these is read as a $\mathrm{C} \times \mathrm{N}$ array, where $C$ is the number of coded behaviors in columns and $\mathrm{N}$ is the number of time blocks in rows, with $\mathrm{C}$ and $\mathrm{N}$ input in lines 40 and 50, respectively. A REM statement in line 130 allows a record of which columns contain which coded behaviors for each interactant. The program is currently set up to read the four maternal behaviors and four infant behaviors detailed earlier in this paper. It has been used with 1,0 dichotomous codes but can also read polytonous codes within each behavioral category with no modifications.

Recoding Data. TLAG analyzes the $2 \times 2$ contingency tables of the dichotomous variables $M(N)$ and $B(N)$, respectively representing maternal and infant change or no change in behavior. The IF statements in lines 160-230 designate the information from the data file that defines these changes in behavior. The IF statements are currently set to give $M(N)$ a value of 1 when any of the mother's coded behaviors change from the previous second and a value of 0 when there is no change. Similarly, $B(N)$ is given a value of 1 for any infant change in behavior and 0 for no change. The values of $M(N)$ and $B(N)$ can be functions of different codes to address many other theoretically relevant research questions. For example, in studies of imitation, data from the maternal and infant smiling codes would go into the recoding procedure to investigate the sequential relations of mother and infant change in smiling behavior.

Analysis of $2 \times 2$ Contingency Tables. The recoded streams of $M(N)$ and $B(N)$ are analyzed in a number of different ways to obtain meaningful measures of sequen-

LISTING 1

The TLAG Program Written in IBM-BASIC for Calculating Lag Sequential Statistics on Dyadic Time Interval Data

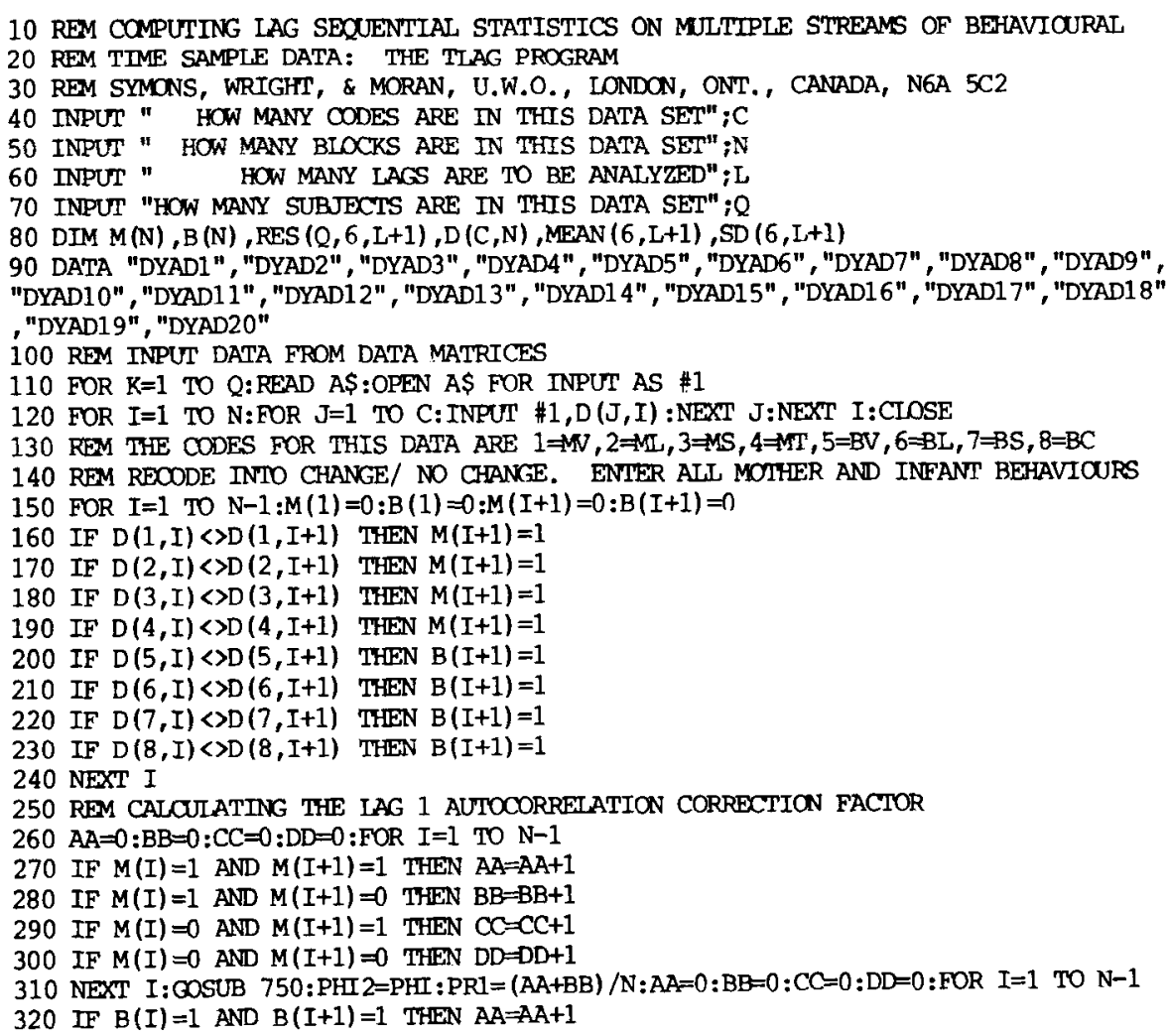




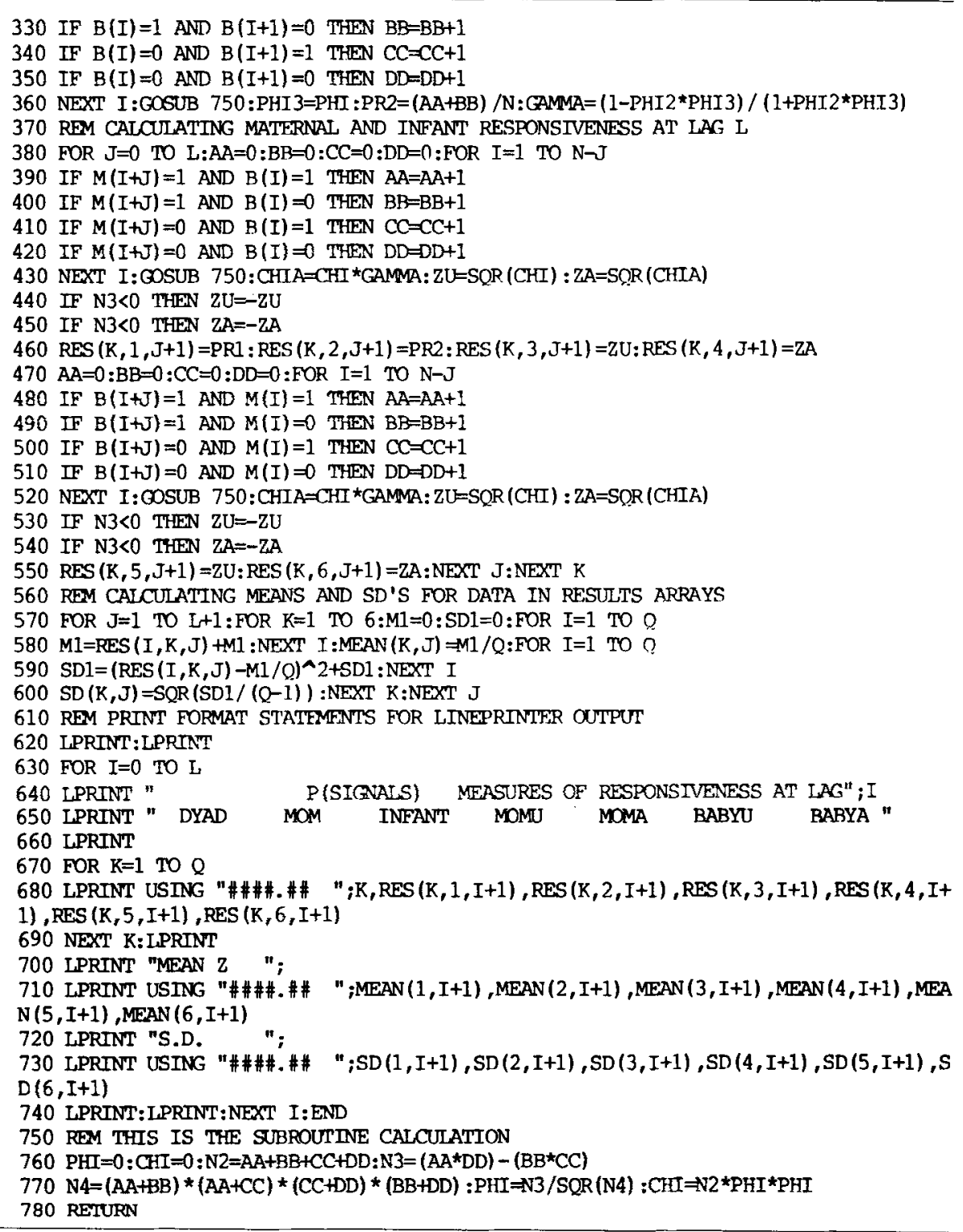

tial dependency between interactants. A number of analyses consist of chi-square statistics based on $2 \times 2$ contingency tables, so these calculations are conducted in a subroutine in lines 750-780.

First, measures of autocorrelation are determined at lag 1 for $M(N)$ data and $B(N)$ data in lines 250-360. These measures are used to calculate the correction for autocorrelation, GAMMA, by which all subsequent chisquare measures of sequential dependency between interactants are multiplied.

Second, measures of sequential dependency at $L$ lags (input in line 60) are calculated. Sequential measures are of both mother at time $T$ versus infant at time $T+L$ (infant responsiveness in lines 480-510) and infant at time $\mathrm{T}$ versus mother at time $\mathrm{T}+\mathrm{L}$ (maternal responsiveness in lines 390-410). Measures saved are $z$ scores, unadjusted and adjusted for autocorrelation.
Compiling and Printing Information. TLAG allows the analysis of $\mathrm{Q}$ data sets (input in line 70; matches the number of data sets listed in line 90 ). For each data set, an LPRINT statement outputs the dyad number, the base rates of mother and infant change in behavior, and unadjusted and adjusted $z$ scores for co-occurrence and maternal and infant responsiveness at $L$ lags. The means and standard deviations of each of these variables are calculated in lines 560-600, and printing instructions are given in lines 610-740.

Other print formats and statements can be utilized to output additional relevant information, such as measures of autocorrelation or actual cell values of the $2 \times 2$ contingency tables. The output modality is currently the line printer, but minor modifications can send output to either the computer screen or an output file using PRINT or WRITE statements, respectively. 


\section{Availability}

The authors have prepared a 5.25-in. flexible disk that contains the TLAG program and dummy data sets to illustrate its use. This disk can be obtained along with documentation by sending a disk and $\$ 5$ for postage and handling to: Douglas K. Symons, Department of Psychology, University of Western Ontario, London, ON N6A 5C2, Canada.

\section{REFERENCES}

Aluson, P. D., \& LiKER, J. K. (1982). Analyzing sequential categorical data on dyadic interaction: A comment on Gottman. Psychological Bulletin, 91, 393-403.

AltmanN, J. (1974). Observational study of behavior: Sampling methods. Behavior, 49, 227-267.

Bakeman, R. (1983). Computing lag sequential statistics: The ELAG program. Behavior Research Methods \& Instrumentation, 15, 330-335.

Bakeman, R., \& GotTman, J. (1986). Observing interaction: An introduction to sequential analysis. New York: Cambridge University Press.

Dumas, J. E. (1986). Controlling for autocorrelation in social interaction analysis. Psychological Bulletin, 100, 125-127.

GARDNER, W. (1987, April). Analyzing sequential categorical data: Individual variation in Markov chains. Paper presented at the biennial meeting of the Society for Research in Child Development, Baltimore, MD.
GARDNER, W., \& HartmanN, D. P. (1984). On Markov dependence in the analysis of social interaction. Behavioral Assessment, 6, 229-236.

Gardner, W., Hartmann, D. P., \& Mitchell, C. (1982). The effects of serial dependence on the use of $\chi^{2}$ for analyzing sequential data. Behavioral Assessment, 4, 75-82.

Moran, G., Krupka, A., Tutton, A., \& Symons, D. (1987). Patterns of maternal and infant imitation during play. Infant Behavior \& Development, 10, 477-491.

MoRAN, G., \& Symons, D. (1987, April). Using sequential analysis to quantify maternal responsiveness in early mother-infant interactions. Paper presented at the biennial meeting of the Society for Research in Child Development, Baltimore, MD.

SACKETT, G. P. (1979). The lag sequential analysis of contingency and cyclicity in behavioral interacton research. In J. D. Osofsky (Ed.), Handbook of infant development (pp. 623-649). New York: Wiley. Sackett, G. P., Holm, R., Crowley, C., \& Henkins, A. (1979). A FORTRAN program for lag sequential analysis of contingency and cyclicity in behavioral interaction data. Behavior Research Methods \& Instrumentation, 11, 366-378.

Simpson, M. J. A. (1979). Problems of recording behavioral data by keyboard. In M. E. Lamb, S. J. Suomi, \& G. R. Stephensen (Eds.), Social interaction analysis: Methodological issues (pp. 137-156). Madison, WI: University of Wisconsin Press.

SYMONS, D. K., \& MORAN, G. (1987). The behavioral dynamics of mutual responsiveness in early face-to-face mother-infant interactions. Child Development, 58, 1488-1495.

(Revision accepted for publication November 17, 1987.) 